\title{
Arthroscopic versus open Latarjet in the treatment of recurrent anterior shoulder instability: a prospective study
}

\author{
Alfredo Savo ${ }^{1}$ \\ Riccardo Maria Lanzetti ${ }^{2}$ \\ Domenico Topa ${ }^{2}$ \\ Vincenzo Campagna ${ }^{2}$ \\ Ferdinando Pulcinelli ${ }^{1}$ \\ Marco Spoliti² \\ 1 Department of Orthopaedics and Traumatology, \\ University of Rome "Tor Vergata" School of Medicine, \\ Rome, Italy \\ 2 Department of Orthopaedics and Traumatology, \\ San Camillo-Forlanini Hospital, Rome, Italy
}

\author{
Corresponding author: \\ Riccardo Maria Lanzetti \\ Department of Orthopaedics and Traumatology \\ "San Camillo-Forlanini" Hospital \\ Circonvallazione Gianicolense 87 \\ 00152 Rome, Italy \\ E-mail: riccardolanzetti@gmail.com
}

\section{Summary}

Introduction: To compare the clinical, radiological parameters and the operation time between the open and the arthroscopic Laterjet procedure.

Methods: 65 patients were prospectively enrolled and divided in 2 groups: Group A, open Latarjet; Group B arthroscopic Latarjet. The mean followup was $26+/-2$ months.

All patients were assessed pre-operatively and at final follow-up with: ROWE, DASH, VAS, ROM and physical examination and with computed tomography.

The operation length was recorded.

Results: At the final follow-up no significative differences was found in ROWE, DASH, VAS, ROM and physical examination.

The mean operation time was 119.94 minutes in the Group A and 217.8 minutes in the Group B $(P<0.005)$.

At CT Scan the orientation of the screw was $27.1^{\circ}$ in the Group $A ; 21.9^{\circ} \pm 5.5^{\circ}$ in the Group $B$ $(P<0.005)$. No statistical difference was found ana- lyzing the angles of the two screws and the distance between the tip of the screw and the border of the glenoid.

There was a decrease of the inferior surface of the glenoid area of $82.3 \mathrm{~mm}^{2}$ in the Group $A$ and $54.5 \mathrm{~mm}^{2}$ in the Group B.

Conclusion: In conclusion, this study may suggest that arthroscopic approach and open procedure are similar in terms of surgical and clinical results.

Level of evidence: III.

KEY WORDS: arthroscopic latarjet stabilisation, coracoid transfer, open latarjet stabilisation, shoulder instability.

\section{Introduction}

Latarjet coracoid bone block, using either one screws or two screws or two screws and a mini plate, represent the most common technique in the treatment of anterior shoulder instability accompanied by bone lesion ${ }^{1}$.

Although the open technique remains the gold standard procedure even today ${ }^{2}$ it was an increasing of the number of arthroscopic Latarjet stabilizations when Lafosse ${ }^{3}$ in 2007 described the possibility of performing the Latarjet procedure via arthroscopy combining the advantages of arthroscopy with those of an open Latarjet ${ }^{4,5}$.

However, some Authors have pointed out that the arthroscopic approach can increase the difficulty and risk of optimal positioning of the bone graft ${ }^{6}$.

Proper coracoid bone graft position and its fusion seem to be one of the keys stabilizing factors ${ }^{7}$.

Several Authors have already reported radiographic evaluation of bone block position after open stabilization; yet, there are very few studies assessing the graft position and fusion post arthroscopic technique 8-11. $^{\text {. }}$

Our hypothesis was that the clinical and radiological results after arthroscopic stabilization are similar of the results achieved after open stabilization.

The aim of this study was to compare the clinical and radiological (Ct scan) outcomes of the arthroscopic and the open Laterjet procedure.

We also evaluated and compared the time required to finish the operation in both surgical technique. 


\section{Materials and methods}

From April 2013 to April 201565 patients with the indication of Latarjet procedure were prospectively enrolled in the present study. All the patients gave the informed consent prior being included into the study. The study was authorized by the local ethical committee and was performed in accordance with the ethical standards of the 1975 Declaration of Helsinki as revised in $2000^{12}$.

From the original 65 patients 60 met the inclusion criteria and were available for the follow-up. These 60 patients were divided in 2 groups: Group A (30 patients, mean age 30-years-old) treated with open Latarjet; Group B (30 patients, mean age 29 years old) treated with arthroscopic Latarjet.

The mean follow-up was $26+/-2$ months.

All patients had chronic, post-traumatic, anterior shoulder instability.

Inclusion criteria:

- $\quad$ age $>17$ and $<45$ years

- symptomatic, unilateral anterior shoulder instability undergoing primary surgery.

Exclusion criteria:

- concomitant rotator cuff lesion

- previous surgery for recurrent anteroinferior dislocation

- multidirectional instability or any contraindication to the surgical intervention.

All patients had positive preoperative apprehension and relocation tests, $<20 \%$ glenoid defect on pre-op CT coronal view.

All the patients also had a standard preoperative range of motion, with a mean active forward elevation of $170.8 \pm 9.8^{\circ}$ and mean external rotation with the arm at the side of $45.0 \pm 17.9^{\circ}$. Only 2 patients $(10.0 \%)$ had an external rotation exceeding $85^{\circ}$.

All diagnoses of chronic shoulder instability, deci- sions to operate and the intervention itself were made by the same orthopedic surgeon, in accordance with the latest guidelines.

Previous shoulder surgery, pathology in the opposite shoulder, history of injury, direction and degree of instability, duration of symptoms, minimum activity level to trigger instability symptoms, and the number of dislocations were also recorded.

\section{Open Latarjet Procedure}

The surgery took place under general anesthesia and with the patient in the sitting position. A sub coracoid approach was performed and the cephalic vein was identified and tilted outwards. After resection of the pectoralis minor tendon the coracoid was exposed and the surgeon performed an osteotomy of the terminal 2$\mathrm{cm}$ of the coracoid process. This was decorticated in its inner aspect and two holes were done in its apophysis. The surgeon made an incision in of the subscapular tendon, the so-called subscapular split and through the aid of a retractor, the head of the humerus was tilted aside. After decortication of the glenoid neck, the coracoid bone graft was transplanted along with the conjoined tendon to the anteroinferior surface of glenoid neck. To reach a good amount of compression between the graft and the glenoid rim, 2 bicortical cannulated screws were used (Fig. 1).

The coracoid and the capsule were then sutured together. The closure was performed layer by layer and then the skin was closed with continuous suture.

\section{Arthroscopic Latarjet Procedure (ALP)}

The patient was positioned in the beach chair position. A diagnostic shoulder arthroscopic evaluation was done, and then attention was turned to antero-inferior glenoid preparation by removing the labrum and the capsule in order to prepare for graft placement. After initial glenoid preparation, through the anterolat-
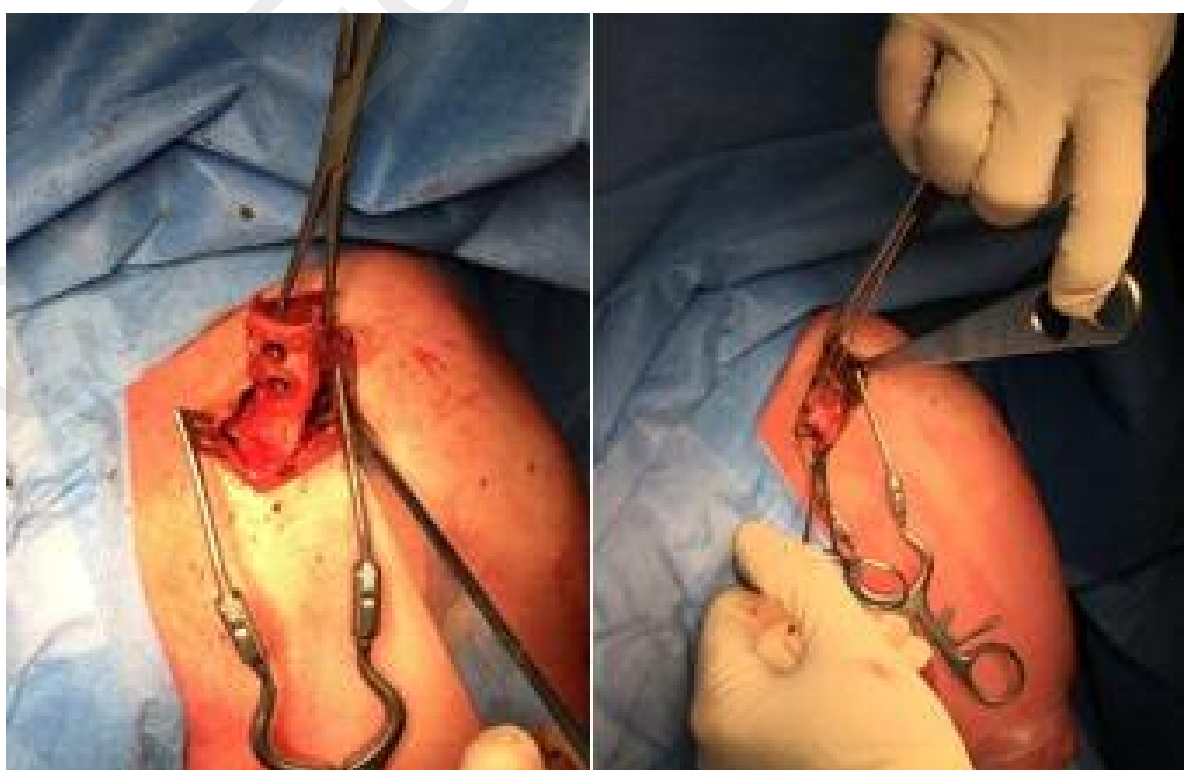

Figure 1. Coracoid harvesting and transplantation in the open procedure. 
eral portal, the rotator interval was opened to reach the coracoid and for the exposure of the anterior and posterior subscapularis.

Inferolateral, inferior and medial portals were made for the coracoid process harvest.

When the coracoid was completely exposed, above the coracoid was created the portal designed for predrilling the coracoid osteotomy (Fig. 2).

A subscapularis split between the middle third and inferior third of the tendon was performed through the electrocautery.

After a gentle blurring of the opposing surfaces of the coracoid graft and the glenoid, the graft was relocated into the appropriate point on the glenoid.

In the final step of the coracoid fixation, the graft was positioned through two K-wires that drilled the graft, the glenoid and the posterior shoulder skin. Two holes were then performed and two screws, one superior and one inferior, were then inserted. The Kwires were then removed and closures of the skin of the portal accesses were performed.

\section{Operative time}

The operation length was recorded in order to calculate the mean operative time and the standard deviation of the arthroscopic technique and the open approach. Time was recorded from the first incision until the suture and the medications were finished. Values obtained from the two operative techniques were then compared between each other.

\section{Rehabilitation}

All patients underwent the same rehabilitation program, with home visits or hospital visits from a stateregistered physiotherapist. In the first 20 days, the shoulder was rested, with the arm blocked tightly to the thorax and only passive elbow, finger and wrist movements were allowed. From day 21 to day 45 , each patient received three to four physiotherapy sessions per week having the purpose of recovering ROM except for the external rotation that was not allowed. After one month and a half, active work and external rotation were started.

\section{Clinical evaluation}

All patients were assessed with:

- ROWE (preoperatively and at final follow-up)

- DASH (preoperatively and at final follow-up)

- VAS (at 3 days, 1 month and at final follow-up)

- ROM (at 3 days, 1 month and at final follow-up)

- Physical examination (at 3 days, 1 month and at final follow-up).

\section{CT analysis}

A computed tomography scan evaluation with 3-dimensional reconstruction of the affected shoulder was performed at final follow-up from surgery in order to evaluate the coracoid bone graft $^{13}$.

A specialist radiologist evaluated the difference in position of the screws and the graft and statistical differences were evaluated.

The Pico method, the oblique sagittal plane for "en face" glenoid views, was used for measurements because it allows a direct view of the glenoid fossa ${ }^{14}$. Manual approximation of the inferior glenoid surface was performed through the estimation of a true circle calculated as described by Sugaya et al. ${ }^{15}$. Once the calculation was made, it was compared to the healthy contralateral. Furthermore, the angle between the two screws and the angle between the screws and the glenoid surface were calculated. Finally, we measured the distance between the glenoid and the tip of the screws; this should not exceed $2 \mathrm{~cm}$ in order to avoid suprascascapular nerve damage (Figs. 3, 4).

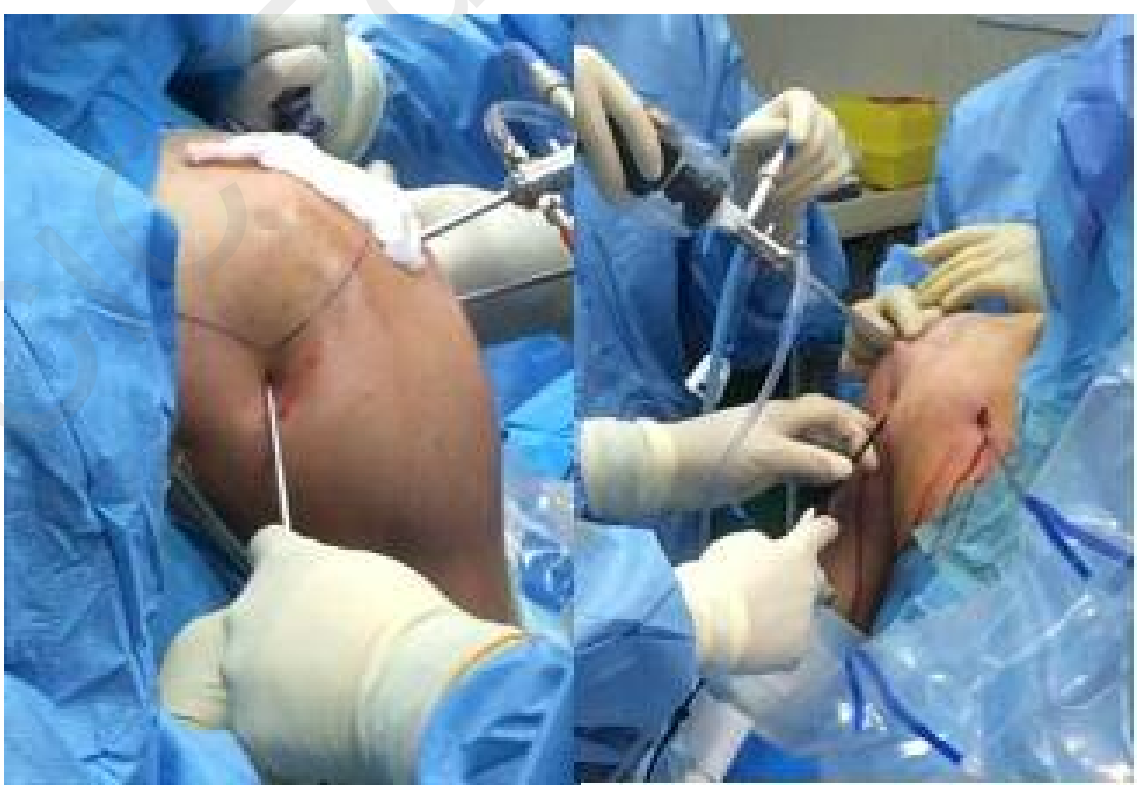

Figure 2. Portals used for the arthroscopic Latarjet procedure. 


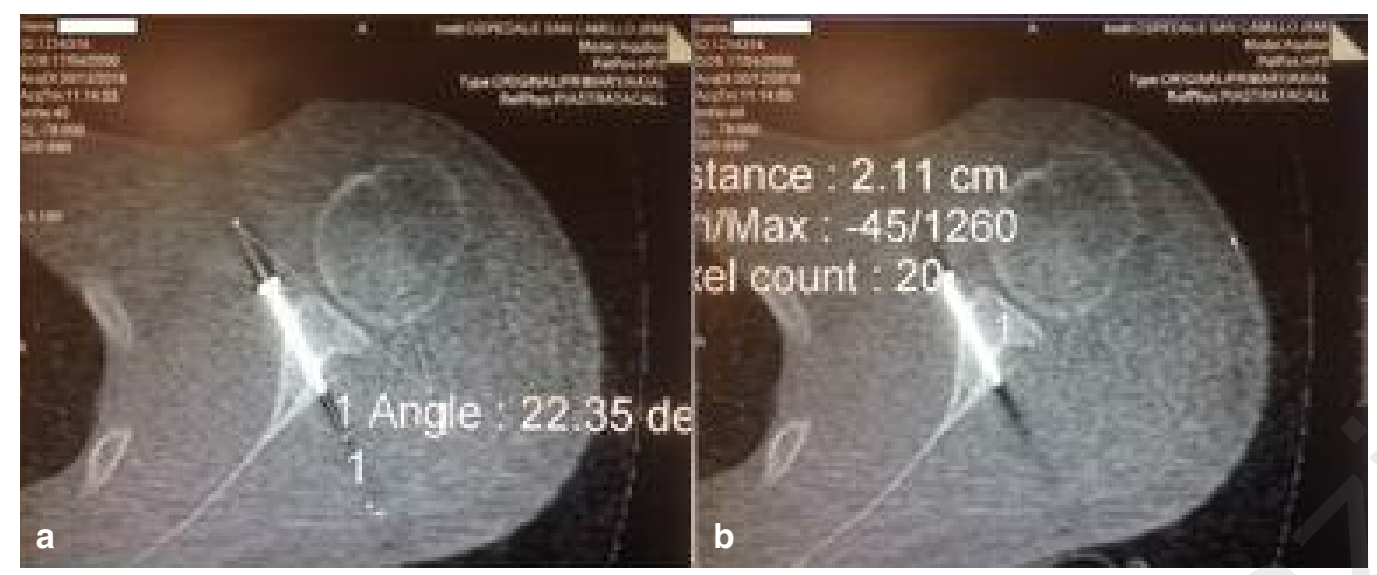

Figure 3. The screw orientation was evaluated on the axial CT scan measuring (a) the angle between the axis of the screw and the glenoid fossa and (b) the distance between the tip of the screw and the border of the glenoid cavity.
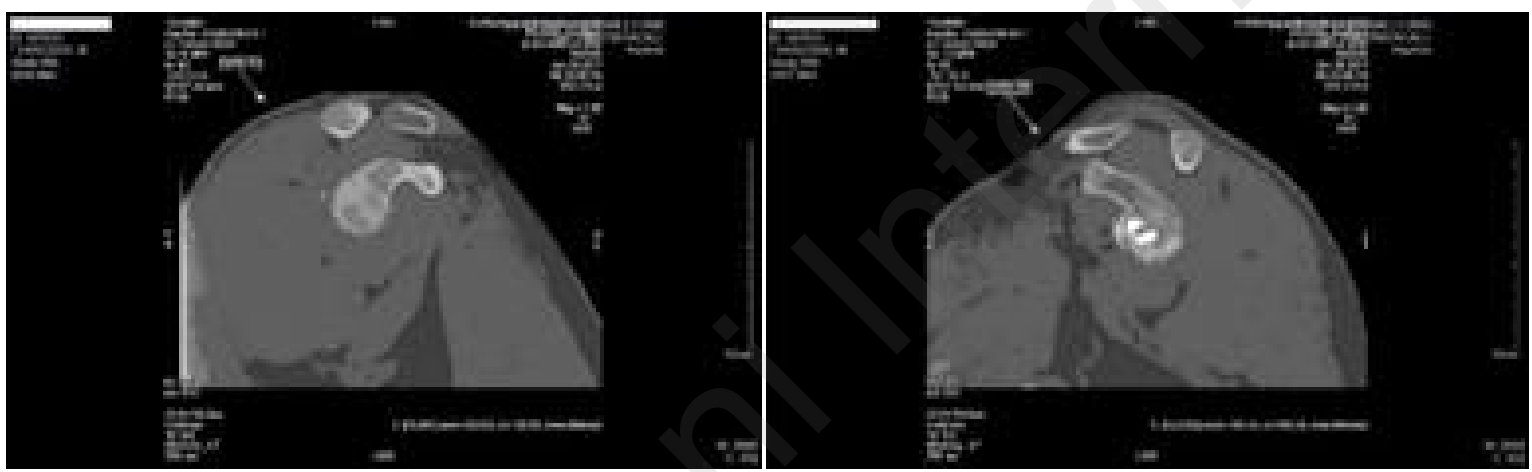

Figure 4. The area of the healthy controlateral glenoid was compared with the affected post-operative glenoid with the CT scan using the Pico meth.

\section{Statistical analysis}

All data were collected in order to present descriptive and analytical statistics. Continuous data were reported as mean and standard deviation. All data evaluated for statistical analysis were checked for normality. In order to compare the various groups for all the selected variables the Student's $t$ test for independent samples was used. Significance level a was set at 0.05 so that for all analysis $p<0.05$ was considered to be statistically significant. A paired-sample $t$ test was used to analyze the difference between the preoperative and post-operative Rowe score. All the statistical analyses were performed with SPSS ver. 10.0 (IBM technologies, 2012).

\section{Results}

The mean time required to finish surgery was $217.8 \pm 35.4$ minutes in the arthroscopic Latarjet group compared with $119.94 \pm 51.8$ minutes in the open Latarjet group. The difference was statistically significant $($ mean difference $=-92.80 ; 95 \% \mathrm{Cl}=-132.99$, 52.60; $P=.000)$.

Just one case that underwent arthroscopic Latarjet operation reported recurrent dislocation or subluxation occurring following traumas. The apprehension test and the relocation test were negative in all patients except for the above-mentioned case. At the final follow-up the mean forward elevation was found to be $160.4 \pm 5.9^{\circ}$ for the open Latarjet group and $160.7 \pm 4.1^{\circ}$ for the arthroscopic Latarjet group, external rotation was found to be $50.1 \pm 14.3^{\circ}$ for the open Latarjet cases and $47.4 \pm 17^{\circ}$ for the arthroscopic Latarjet.

The post-operative Rowe score in the open Latarjet was found to be $79.50 \pm 16.06$ compared with the $81.00 \pm 21.05$ of the arthroscopic treatment. No significant difference (mean difference $=-1.50 ; 95 \% \mathrm{Cl}=$ $19.09,16.09 ; P=.860$ ) was found in the two groups. In both the arthroscopic and the open Latarjet group, Rowe score was significantly improved after surgery (Tab. I).

Comparing the two groups in relation to the Dash score, we didn't find any statistical difference (Tab. I). On the contrary, the pain recorded in the first three days following the operation turn out to be significantly better (mean difference $=25.50 ; 95 \% \mathrm{Cl}=17.15$, $33.85 ; \mathrm{P}=.000$ ) in the arthroscopic group (mean, $29.50 \pm 7.25)$ than in the open (mean $55.00 \pm 10.30$ ). 
Table I. Rowe and Dash results.

\begin{tabular}{lllll}
\hline & Preoperative & & Postoperative \\
\hline & Group A & Group B & Group A & Group B \\
\hline Rowe & $30.3 \pm 7.2$ & $29 \pm 8.2$ & $79.50 \pm 16.06$ & $81.00 \pm 21.05$ \\
Dash & $72.9 \pm 5.8$ & $74 \pm 6.3$ & $6.3 \pm 4.2$ & $8.4 \pm 5.2$ \\
\hline
\end{tabular}

Also regarding the pain sensed at the 1 month followup, it turns out to be statistically different (mean difference $=09.00 ; 95 \% \mathrm{Cl}=2.17,15.82 ; \mathrm{P}=.013)$ in favor of arthroscopic patients (mean $17 \pm 7.15$ ) respect to the open ones (mean, 26.00 \pm 7.39 ). However at the final follow-up there were no differences in VAS score between the two groups.

Additionally, patient of the open Latarjet yielded statistically greater (mean difference $=-1.40 ; 95 \% \mathrm{Cl}=-$ $2.11,-.68 ; \mathrm{P}=.001$ ) time to recovery than the arthroscopic Latarjet procedure, the former having a mean of $3.4 \pm 0.84$ out of 4 , respect to the latter mean of $2.00 \pm 0.67$ out of 4 .

Concerning the wound, results reported again a statistical difference (Mean difference $=-1.30 ; 95 \% \mathrm{Cl}=$ $-2.00,-.59 ; P=.001)$ in the subjective happiness in favour of the arthroscopic yielding a mean of $3.8 \pm 0.63$ out of 4 , in contrast to the open approach that scored a mean of $2.5 \pm 0.85$ out of 4 .

Finally, also the subjective satisfaction of the quality of life after the intervention reported a statistically significant difference $($ mean difference $=-.90 ; 95 \% \mathrm{Cl}=$ $-1.66,-.14 ; P=.022)$ between the two groups, having the arthroscopic a mean of $3.70 \pm 0.49$ out of 4 with respect of the open mean of $2.80 \pm 1.03$ out of 4 .

The CT scan revealed that all the transferred coracoid processes were found to be level with the articular surface of the glenoid in all patients of both groups, except for one. Coracoid grafts were below the equator in $90 \%$ of patients both in the open arthroscopic group. There was no significant difference between the 2 groups.

The orientation of the screw was $27.1 \pm 5.2^{\circ}$ in the open group compared with $21.9 \pm 5.5^{\circ}$ in the arthroscopic group. The difference was statistically significant (mean difference $=5.23 ; 95 \% \mathrm{Cl}=.17,10.28 ; \mathrm{P}$ $=.043$ ). Analyzing the angles formed by the two screws, in the arthroscopic group the mean outcome found was $2.20^{\circ} \pm 4.24$ while in the open was $2.60 \pm 3.44$. No statistical difference was found (mean difference $=.40 ; 95 \% \mathrm{Cl}=-3.22,4.02 ; \mathrm{P}=.819$ ).

The distance between the tip of the screw and the border of the glenoid was $2.1 \pm 0.3 \mathrm{~cm}$ for the arthroscopic group and $2.2 \pm 0.3 \mathrm{~cm}$ for the open group. There was no significant difference (mean difference $=.12 ; 95 \% \mathrm{Cl}=.20, .44 ; \mathrm{P}=.448)$.

After the reconstruction, there was a decrease of the inferior surface of the glenoid area, calculated comparing it to the healthy contralateral, of $54.5 \pm 25.4$ $\mathrm{mm}^{2}$ (range, 5 to $130 \mathrm{~mm}^{2}$ ) for the arthroscopic group and of $82.3 \pm 32.3 \mathrm{~mm}^{2}$ (range, 8 to $190 \mathrm{~mm}^{2}$ ) for the open group.

Comparing the outcomes of the reductions in the areas of the inferior glenoid surface (calculated by the difference with the contralateral healthy inferior glenoid surface) between the two groups we did not find any statistical difference (mean difference $=2.39$; $95 \% \mathrm{Cl}=-4.90,9.70 ; \mathrm{P}=.499$ ) having the arthroscopic patient a mean of $7.10 \pm 8.65^{\circ}$ reduction and the open group a mean of $9.50^{\circ} \pm 6.79$ of reduction.

\section{Discussion}

This study confirmed our initial hypothesis: the clinical and radiological results after arthroscopic stabilisation are similar of the results achieved after open stabilisation in Latarjet procedure.

The first finding of our study regarded the time length of the surgery, as expected we found that arthroscopic approach requires greatly increased time in respect to the open procedure.

Concerning the clinical results, functional test as ROM analysis, apprehension test, relocation test and Rowe score showed that there was no statistical difference between the two groups sampled for our study. Regardless of the surgical approach adopted, the post-operative Rowe score improved in both groups when compared to baseline pre-operative score, thus showing that Latarjet procedure offers a clear benefit to patients affected by shoulder instability.

Regarding the pain felt by the patient in the first 3 days after the operation and the pain perceived during the first month there was a considerable difference in favour of the arthroscopic technique.

Arthroscopic technique also revealed to shorten the time for the entire recovery and a prompter return to routine activities.

Additionally, both the surgical wound satisfaction and the quality of life following the treatment were found to be superior in the arthroscopic group.

The radiographic outcomes outlined that there was no statistical difference in the 2 groups regarding the reconstructed area following the operation. Neither in the coracoid graft positioning nor in the screws angle, nor in the distance between the tip of the screw and the border of the glenoid surface was found any difference in the open and arthroscopic group.

Finally, it was found that the only radiographical ele- 
ment showing a statistically significant difference between the two groups concerned the angle between the glenoid surface and the head of the screws.

In Latarjet procedure, successful treatment is influenced by many factors but one of the most important is the accurate placement of the coracoid graft. Besides the correct positioning of the graft onto the glenoid neck it is of paramount importance that the screws are placed parallel to each other, perpendicular to the glenoid neck and precisely at the center of the glenoid defect ${ }^{16}$.

It can be stated that with an anterior arthroscopic approach it is still not possible an adequate positioning of the coracoid graft. This may be due to the fact that the anterior arthroscopic approach bears an intrinsic difficulty in drilling completely perpendicular holes in the glenoid defect during the preparation phase.

To the best of our knowledge, the only study that seems to achieve a total perpendicular approach to the glenoid defect was the one performed by Taverna et al. in which a posterior arthroscopic Latarjet technique is described ${ }^{16}$.

The better parallelism in the study of Taverna et al. is due to the fact that when the glenoid surface, normally antiverted of $20^{\circ}$, is approached anteriorly there is an interference of vital structures such that a complete perpendicular drilling is impossible. For this reason, it seems reasonable that a posterior approach could confer better results in term of parallelism and in our opinion its evaluation should be further investigated in future studies.

Moreover, our findings showed that just one case that underwent arthroscopic Latarjet operation, reported recurrent dislocation at of 2 years' follow-up and no significant restriction of patients' range of motion was found. This result is in line with literature ${ }^{5,17-19}$.

The comparison between arthroscopic and open Latarjet is debated among orthopedic surgeons. Very few studies are available comparing those two techniques in regard of both clinical and radiological outcomes. The initial assumption was that the two procedures were not equivalent because of the advantage of using an arthroscopic approach for Latarjet, in particular smaller incision, less soft-tissue dissection, better visualization of the joint, better repair accessibility, and the potential shorter time for full recovery. Regardless of this appealing aspect, there is a lack of evidence to support this hypothesis. Our study shows that results are similar but arthroscopic technique can give some advantages as subjective satisfaction of the quality of life after the intervention but with longer surgical time.

This study has some limitations: first, the follow-up time was relatively short, and the long-term effect of this modified arthroscopic Latarjet procedure on the degenerative changes of the glenohumeral joint cannot be evaluated. Third, the patients' return to sports or work was not documented in the final evaluation. Finally the rate of nonunion was not reported.

In conclusion, this study may suggest that arthroscopic approach and open procedure are similar in terms of surgical and clinical results. As expected, the arthroscopic surgery may give some advantages in terms of post-operatory pain and aesthetic results. Moreover, it shows that both the open and the arthroscopic Latarjet procedures can obtain equivalent outcomes except for shoulder functional recovery timing and for the surgical wound satisfaction. Maybe it is necessary a detailed consultation with the patient showing benefits and dangers of each procedure before choosing.

It is desirable that studies with randomized arms and larger samples will be conducted in the future in order to draw solid conclusions on this topic.

\section{References}

1. Mascarenhas R, Rusen J, Saltzman BM, et al. Management of humeral and glenoid bone loss in recurrent glenohumeral instability. Adv Orthop. 2014;2014:640952. doi: 10.1155/2 014/640952.

2. Bhatia S, Frank RM, Ghodadra NS, et al. The outcomes and surgical techniques of the latarjet procedure. Arthroscopy. 2014 Feb;30(2):227-235. doi: 10.1016/j.arthro.2013.10.013.

3. Lafosse L, Lejeune E, Bouchard A, Kakuda C, Gobezie R, Kochhar T. The arthroscopic Latarjet procedure for the treatment of anterior shoulder instability. Arthroscopy. $2007 \mathrm{Nov} ; 23$ (11):1242.e1-5.

4. Lafosse L, Boyle S. Arthroscopic Latarjet procedure. J Shoulder Elbow Surg. 2010 Mar;19(2 Suppl):2-12. doi: 10.1016/ j.jse.2009.12.010.

5. Dumont GD, Fogerty S, Rosso C, Lafosse L. The arthroscopic Latarjet procedure for anterior shoulder instability: 5-year minimum follow-up. Am J Sports Med. 2014 Nov;42(11):25602566. doi: $10.1177 / 0363546514544682$.

6. Boileau P, Mercier N, Roussanne Y, Thélu CÉ, Old J. Arthroscopic Bankart-Bristow-Latarjet procedure: the development and early results of a safe and reproducible technique. Arthroscopy. 2010 Nov;26(11):1434-1450. doi: 10.1016/ j.arthro. 2010.07.011.

7. Wellmann M, Petersen W, Zantop T et al. Open shoulder repair of osseous glenoid defects: biomechanical effectiveness of the Latarjet procedure versus a contoured structural bone graft. Am J Sports Med. 2009 Jan;37(1):87-94. doi: 10.1177/ 0363546508326714.

8. Hovelius L, Sandström B, Sundgren K, Saebö M. One hundred eighteen Bristow-Latarjet repairs for recurrent anterior dislocation of the shoulder prospectively followed for fifteen years: study I-clinical results. J Shoulder Elbow Surg. 2004 Sep-Oct;13(5):509-516.

9. Mizuno N, Denard PJ, Raiss P, Melis B, Walch G. Long-term results of the Latarjet procedure for anterior instability of the shoulder. J Shoulder Elbow Surg. 2014 Nov;23(11):16911699. doi: 10.1016/j.jse.2014.02.015.

10. Meyer DC, Moor BK, Gerber C, Ek ET. Accurate coracoid graft placement through use of a drill guide for the Latarjet procedure. J Shoulder Elbow Surg. 2013 May;22(5):701-708. doi: 10.1016/j.jse.2012.06.012.

11. Casabianca L, Gerometta A, Massein A, et al. Graft position and fusion rate following arthroscopic Latarjet. Knee Surg Sports Traumatol Arthrosc. 2016 Feb;24(2):507-512. doi: 10.1007/s00167-015-3551-6.

12. Padulo J, Oliva F, Frizziero A, Maffulli N. Muscles, Ligaments and Tendons Journal - Basic principles and recommendations in clinical and field science research: 2016 update. MLTJ. 2016;6(1):1-5. 
13. Cautiero F, Russo R, Di Pietto F, Sabino G. Computerized tomographic assessment and clinical evaluation in shoulder instability treated with the Latarjet-Patte procedure using one screw and washer. Muscles Ligaments Tendons J. 2017 May 10;7(1):26-33. doi: 10.11138/mltj/2017.7.1.026.

14. Magarelli N, Milano G, Baudi P, et al. Comparison between 2D and 3D computed tomography evaluation of glenoid bone defect in unilateral anterior gleno-humeral instability. Radiol Med. 2012 Feb;117(1):102-111. doi: 10.1007/s11547-011-0712-7.

15. Sugaya H, Moriishi J, Dohi M, Kon Y, Tsuchiya A. Glenoid rim morphology in recurrent anterior glenohumeral instability. $J$ Bone Joint Surg Am. 2003 May;85-A(5):878-884.

16. Taverna E, Ufenast H, Broffoni L, Garavaglia G. Arthroscopically assisted Latarjet procedure: A new surgical approach for accurate coracoid graft placement and compression. Int $\mathrm{J}$
Shoulder Surg. 2013 Jul;7(3):120-123. doi: 10.4103/09736042.

17. De Carli A, Fabbri M, Lanzetti RM, Ciompi A, Gaj E, Beccarini $G$, Vetrano M, Ferretti A. Functional treatment in rotator cuff tears: is it safe and effective? A retrospective comparison with surgical treatment. Muscles Ligaments Tendons J. 2017 May 10;7(1):40-45. doi: 10.11138/mltj/2017.7.1.040.

18. Boileau P, Thélu CÉ, Mercier $\mathrm{N}$ et al. Arthroscopic BristowLatarjet combined with bankart repair restores shoulder stability in patients with glenoid bone loss. Clin Orthop Relat Res. 2014 Aug;472(8):2413-2424. doi: 10.1007/s11999-014-3691-x.

19. Pantalone A, Vanni D, Guelfi M, Di Mauro M, Abate M, Salini V. Arthroscopic treatment of shoulder instability in professional athletes. Muscles Ligaments Tendons J. 2016 Feb 12;6(4): 440-444. doi: 10.11138/mltj/2016.6.4.440. 